\title{
AC 2008-1212: A PROGRAM FOR DISTRIBUTED LABORATORIES IN THE ECE CURRICULUM
}

\section{Bonnie Ferri, Georgia Tech}

Bonnie Heck Ferri received the BS from Electrical Engineering from Notre Dame in 1981, the MS in Mechanical and Aerospace Engineering from Princeton in 1984, and the PhD in Electrical Engineering from Georgia Tech in 1988. She is currently a Professor and Associate Chair for Graduate Affairs in ECE at Georgia Tech. Her research has been in the areas of embedded control systems, applications of control, power electronics, and education. She is the recipient of the 2007 IEEE Education Society Harriet Rigas Award.

\section{Jill Auerbach, Georgia Institute of Technology}

Jill Auerbach is a Senior Academic Professional in the School of Electrical and Computer Engineering at Georgia Institute of Technology. As the Coordinator of Assessment and Student Retention in the School, she is responsible for accreditation and program review requirements and assessment of several special academic programs. In addition, Jill directs programs that promote student retention and success, especially among underrepresented, female and transfer student cohort groups. Her educational background is in the fields of Policy Analysis and Public Administration, with emphasis on research methodology.

\section{Joel Jackson, Georgia Tech}

Dr. Jackson received a dual major B.S. degree in Electrical and Computer Engineering and Chinese from Brigham Young University. The recipient of a National Science Foundation Graduate Fellowship to continue his research at Georgia Tech, he obtained his M.S. degree in 1995 and completed his Ph.D. in 1999. Dr. Jackson is currently involved in developing methods for computer-enhanced education and distance learning in the Center for Signal and Image Processing in the School of ECE at Georgia Tech.

\section{Jennifer Michaels, Georgia Institute of Technology}

Jennifer E. Michaels received her Bachelor's degree in Electrical Engineering from Georgia Tech in 1976, and her M.S. and Ph.D. degrees in Theoretical and Applied Mechanics from Cornell University, in 1982 and 1984, respectively. She was co-founder of JTM Systems and Consulting, Inc., Ithaca, New York, in 1980, working primarily on the development, fabrication and commissioning of custom automated ultrasonic inspection systems. She continued this work from 1988 until 2002 as Manager of Systems Development at Panametrics, Inc., in Waltham, Massachusetts. In 2002 she joined the faculty of the School of Electrical and Computer Engineering at Georgia Tech where she is an Associate Professor. She is co-director of the QUEST (Quantitative Ultrasonic Evaluation, Sensing and Testing) Laboratory at Georgia Tech, where her current research interests are structural health monitoring, nondestructive evaluation and materials characterization.

\section{Douglas Williams, Georgia Tech}

Douglas B. Williams received the BSEE, MS, and $\mathrm{PhD}$ degrees in electrical and computer engineering from Rice University, Houston, Texas, in 1984, 1987, and 1989, respectively. In 1989, he joined the faculty of the School of Electrical and Computer Engineering at the Georgia Institute of Technology, Atlanta, Georgia, where he is currently Professor and Associate Chair for Undergraduate Affairs. There he is also affiliated with the Center for Signal and Image Processing and the Arbutus Center for Distributed Engineering Education. 


\title{
A Program for Distributed Laboratories in the ECE Curriculum
}

\begin{abstract}
This paper describes a project that seeks to improve undergraduate learning by developing a cohesive program where experiments are introduced into a wide selection of ECE courses that currently do not have labs. Most of the experiments are low cost and portable, which facilitates a decentralized laboratory environment where students perform the experiments at their homes or in the classroom rather than in dedicated laboratories. We will assess our work through the involvement of twelve faculty members teaching ten courses, some of which are offered in distance learning settings.
\end{abstract}

\section{Introduction}

Experiments are vital to the understanding of basic principles of science and engineering, yet experiments are generally relegated to laboratory courses conducted in centralized laboratories containing expensive equipment. Another model, discussed here, is to build low-cost experiments that can be used in the classroom or at home by students. Recent advances in embedded processors and in sensor technology makes it possible to develop low-cost processorbased experiments that are portable enough for this purpose. Another source of readily available low-cost experiments are those sold through hobby outlets and for K-12 educational purposes, both of which could be used to demonstrate fundamental concepts in science and engineering when augmented with high level explanations and projects suitable for college-level students. At present, the fidelity of the results obtained from existing low-cost embedded processor platforms and simple experiments is not at the level needed for typical lab-based courses, but they can make a big contribution as a supplement to lecture-based courses.

Eventually, embedded technology may replace the need for large, expensive laboratory equipment in centralized labs. As such, this program may be at the forefront of a trend in engineering curricula toward decentralized laboratories containing portable, embedded processor-based technologies with experiments that are distributed throughout the curriculum. This trend would mirror the curricula changes that took place from 1975 to 2000 surrounding the use of computers in education. Initially, students used mainframes and card readers in centralized locations. Advances in computers facilitated the move to decentralized computer labs. The most recent move, which is still in progress, is towards student ownership of PCs along with all of the course software. The program discussed in this paper initiates the move from expensive, centralized experimental laboratories and dedicated lab courses towards low-cost decentralized laboratories, where even personal ownership of laboratory platforms may occur.

The use of experimental demonstrations in lecture-based engineering and science courses is not new; usually, demos are performed by instructors in the class. Recently, there has been development of some hands-on experimental platforms for engineering students to use in the classroom or to take home [1-5]. These efforts generally are done on a small scale, uncoordinated between courses and often without extensive web-based support; however, these papers do demonstrate the feasibility and usefulness of hands-on experiments to supplement lecture courses. 


\section{Experimental Modules}

The wide-spread adoption of a particular demonstration or experimental platform by instructors depends on how simple it is to duplicate and use, how expensive it is, how versatile it is to be used for a variety of projects, and how well the experiment conveys fundamental concepts to students. These considerations are at the forefront of the experimental modules described in this paper.

Different physical experiments are being developed that have different modes of delivery: handson experiments done in class, hands-on experiments to be taken home, hands-on experiments done in a laboratory that is shared among classes, or in-class demonstrations done by the instructor. It is assumed that all of the experiments enhance or demonstrate fundamental or theoretical concepts normally taught in lecture-based courses. These different modes of delivery have the following additional characteristics:

In-class experiments

- Lab groups of 2-3 people

- Experiment can be done on desktops using portable power, if needed, such as batteries

- Length of lab should be less than 50 minutes

- Prelab can be part of the lab

Take-home experiments

- Lab groups of 2-3 people

- Scheduling (1/2 day to 1 day check-out periods)

- Check-out/check-in procedures defined

- Ability to mail the hardware to remote campuses or locations

Lab shared among courses

- Ability to store all portable experiments and be suitable for running the take-home or inclass experiments in the lab

- House expensive equipment for lab-based experiments

- Scheduling of lab time for class projects and coordination among courses

- Dedicated TA or lab support personnel for lab

Instructor-led demonstrations

- Portable enough to be brought into a class

- Uses equipment that is too expensive, fragile, or sophisticated for use by students in that course

Different platforms are being used for the embedded computing experiments: LEGO NXT, LEGO Mindstorm RCX, PIC processors, and the National Instruments Speedy DSP board. A number of tradeoffs exist between the processors [6]; thus, the resulting experiments can have different attributes and capabilities. The LEGO RCX platform has been used for some of the computer engineering experiments, partly because of the open source operating system BrickOS (http://brickos.sourceforge.net/) that was developed for it and the wealth of related experiments already available using it [7]. The LEGO NXT platform improves on the RCX considerably in terms of processor speed, memory, and sensor resolution, so it works well for controls and 
robotics experiments. The PIC processor is chosen for some of the experiments since it is the most widely used 8-bit microcontroller in the world; furthermore, it is cheap and easy to program [8]. The PIC kit 2, for example, has a USB cable for programming from a PC, students can download a free version of the development environment for programming (including a $\mathrm{C}$ compiler), it is easy to use, and it lists for $\$ 49$, making it suitable for take-home use by students.. Low cost DSP boards are used for senior projects in ECE schools, research prototypes, and, even continuing education. They provide an inexpensive (i.e., less than \$500) means of real-time data acquisition and processing. There are several boards, such as the series of Texas Instruments' DSP Student Kits and National Instruments' NI-Speedy DSP, that have been developed specifically for the educational market.

\subsection{Web Component}

The web material developed for each experiment assists new faculty in assimilating the experiments and serves as a resource to students who use the experiments. The web modules have the following components:

- instructional video and detailed directions for building the experiment

- demonstration video of the experiment

- interactive demonstration

- descriptions of sample projects that instructors may assign for the experiment

- tutorial for students describing the fundamental concepts demonstrated

- online quiz that tests the students' knowledge of the fundamental concepts being tested

The format for the online tutorial and test is similar to one developed by Heck in the web component of a textbook [9]. That website includes online demos for a variety of fundamental topics. The format for the demos includes directions, a tutorial to explain the fundamental concepts, and an online quiz. See the sample of an online quiz shown in Figure 1 for the demo on periodicity of sums of sinusoids. The purpose of the quiz is to relate the material in the demo to material that might appear on a test administered in a class. This approach to web-based modules, where the material is directly related to homework and test problems, has proven to increase students' performance on tests [10]. The web modules can be integrated into a course management system so that students' performance on the web modules can be tracked.

One goal of the interactive demo is to give students the ability to try different strategies prior to working on the physical experiment. The interactive demos are relatively complete simulations of the experiments. The demo also allows students to preview the experiment, so that they have an idea of how the experiment should perform in advance, and are able to recognize problems and mistakes quickly. The interactive module also allows the student to review the lab work later when access to the hardware may be difficult or impossible. 


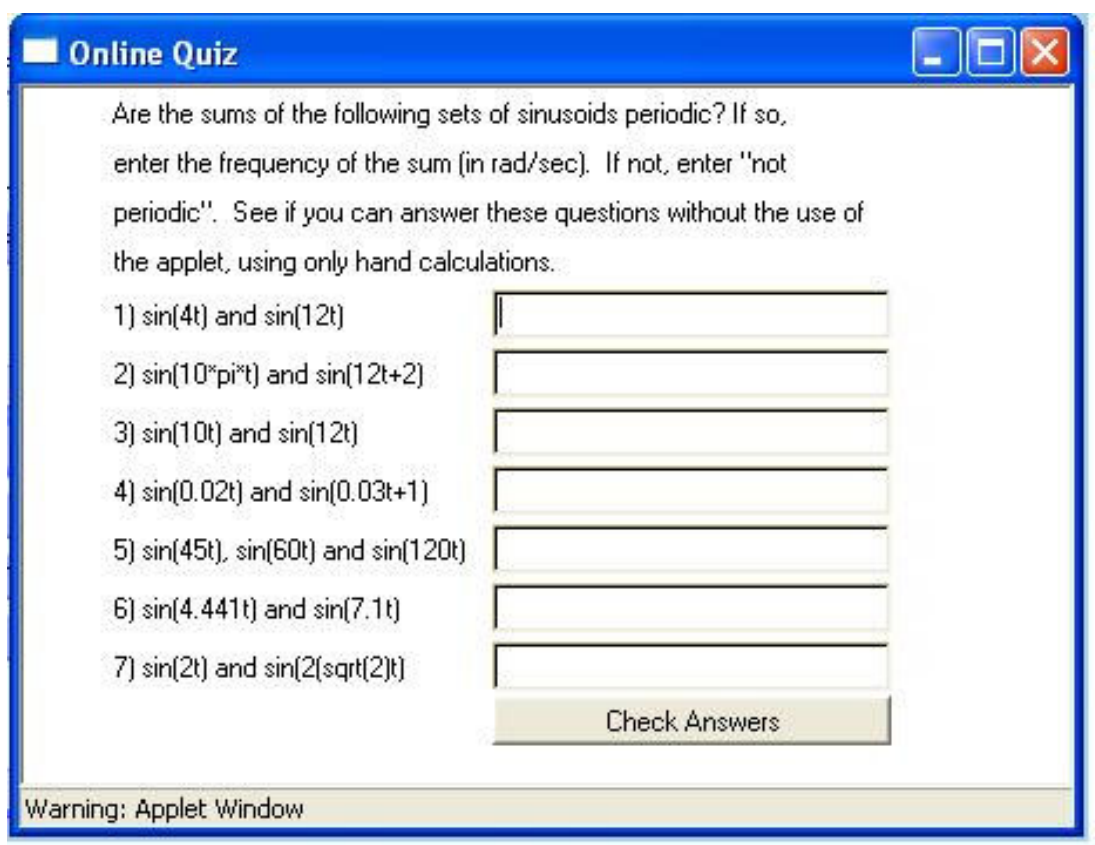

Figure 1: Screen shot of an online quiz from [9] representative of the style being developed for this project. The online quiz gives questions related to the experiment that are similar to test questions on the topic.

To date, five experimental modules have been implemented in classes and several more have been developed. The modules are listed below with the components needed, the mode of delivery, and the fundamental concepts taught.

\subsection{Description of Experiments}

\section{Digital Logic Module}

- Proto-board, batteries, IC chips

- Mode of Delivery: Done in class

- Fundamental Concepts: memory in circuits, sequential logic

State machines are difficult for many students to comprehend because the output depends on a time history of the input, as opposed to combinational circuits that have no time dependence. The experiment serves several purposes: 1) to help students comprehend the dependence on time by having a button that students can push to advance the clock and see how the resulting LED outputs change with different input sequences, 2) to introduce students to protoboards and integrated chips, and 3) to require the students to design and build a physical implementation of a state transition diagram.

The board is given to students partially prewired as shown in Figure 2, with power, clock, and reset buttons connected. The prewiring allows the experiment to be completed in the allotted 50 minute time slot of a lecture course. The students have a prelab where they design a state machine circuit from a given state transition diagram. The lab itself consists of two parts: students must wire a sample state machine circuit, and then they are asked to wire the circuit that they designed. The chips are all CMOS, which require low power, so that the 
boards can be powered by batteries. (The figure shows the battery pack on board.) The cost of this particular model is approximately $\$ 45$. The integrated chips range from $\$ 0.05-\$ 0.50$ each, but the protoboard itself costs about $\$ 40$. There are much cheaper protoboards on the market that could be substituted to reduce costs.

The interactive online demo, which is a simulation of the actual board, gives students the ability to try out their designs and connect the wires in the simulated circuit. If they "blow out" a chip in the simulation, they can get immediate feedback on screen. Mistakes are less time-consuming and costly than with the real experiment. Students would be required to test their prelab design on the simulated circuit prior to coming to class to work on the physical circuit. A screen shot of the protoboard demo is shown in Figure 3.

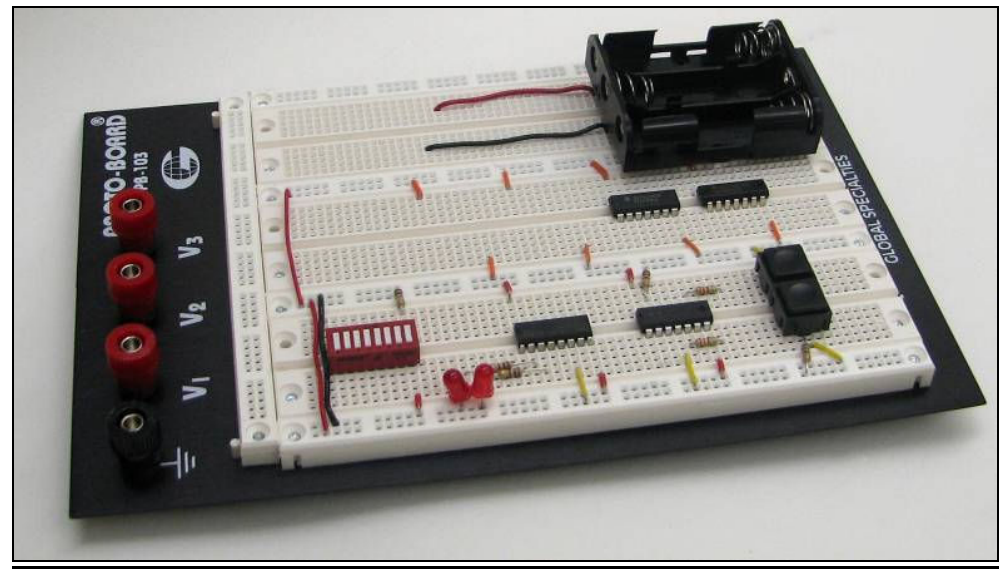

Figure 2: State machine experiment. The protoboard is partially prewired (with power and clock) for students so they can concentrate on the chip interconnections. A push button allows students to advance the clock and see the resulting output of a circuit that they designed and built. 


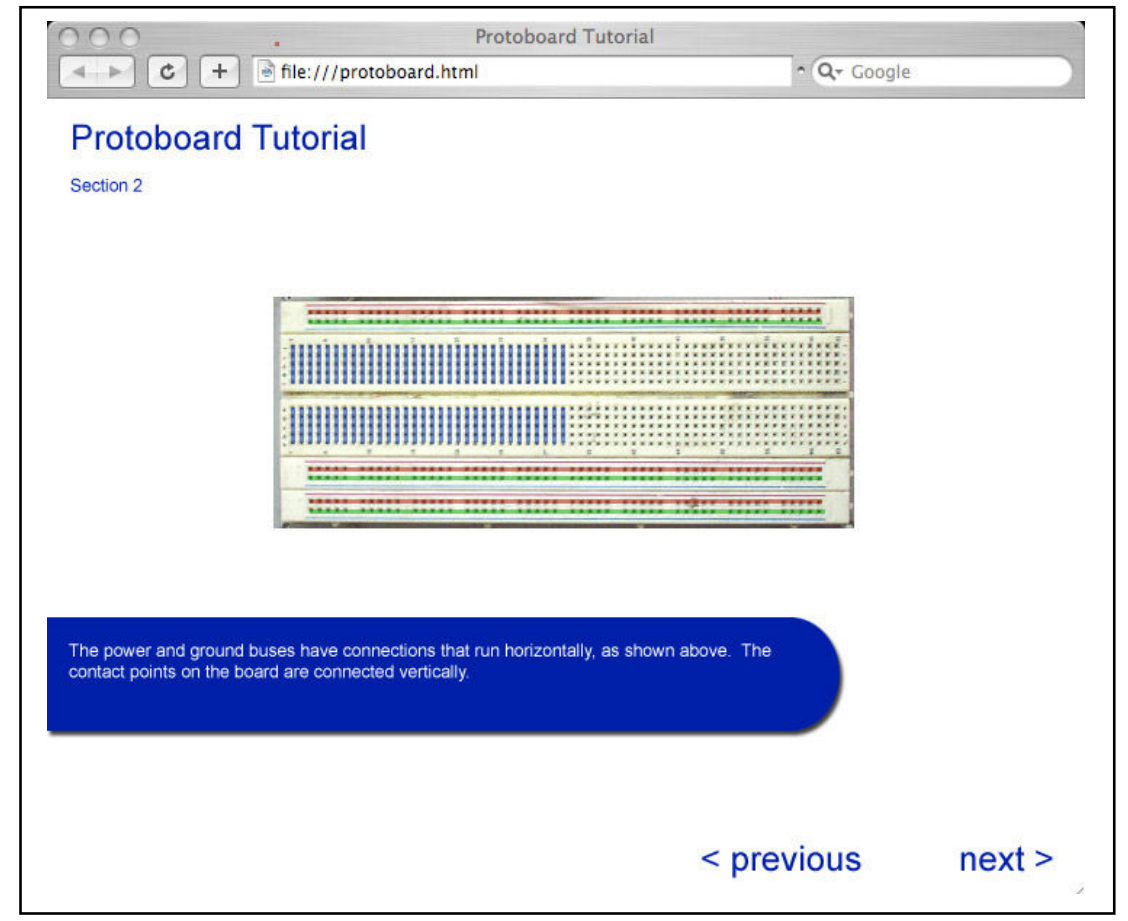

Figure 3: Screen shot of an on-line interactive demo for the use of a virtual protoboard.

\section{Signal Processing Modules}

- LEGO, PIC, and NI Speedy 33 platforms, motor and sensors

- Mode of Delivery: Done at home, in class, or in shared lab

- Fundamental Concepts: Sampling/Aliasing, FIR filters, frequency response

We have used the National Instruments (NI) LabView graphical programming environment to develop several low-cost, real-time experimental modules to supplement projects that are presently done offline and in software as part of our existing DSP classes. Descriptions of these preexisting software projects are available to the general public through a textbook [11]. Class projects examine subjects such as music synthesis based on sinusoidal synthesis or FM synthesis, sampling and aliasing, convolution, and filtering. The goal of the new experimental modules is to supplement the software projects with real-time examples for both classroom demonstration and laboratory experimentation. The flexibility of the hardware platforms and the LabView environment have allowed us to develop new experiments that run in real-time on DSP hardware and thereby complement existing projects that the students currently implement in MATLAB.

Three different hardware platforms were examined for each module. Two are external to the PC - NI's Speedy-DSP Board and the LEGO Mindstorms NXT brick, see Figure 4. The third platform is the PC's internal sound card. For each project, modules were developed for these different hardware platforms that were as similar as possible. However, the LabView software libraries vary somewhat between these platforms. Consequently, not all platforms 
were practical for every project. Where possible, stand-alone programs were developed that do not require the user to own a copy of LabView. Stand-alone applications consist of executable code originally compiled within LabView that can run on the host PC and/or on external hardware.

Modules have been developed for projects that implement:

- Frequency Response of FIR Filters

- Effect of Zero Locations on FIR Filters

- Cascaded Filters

- Suppression of Sinusoidal Interference

- A 7-Band Equalizer
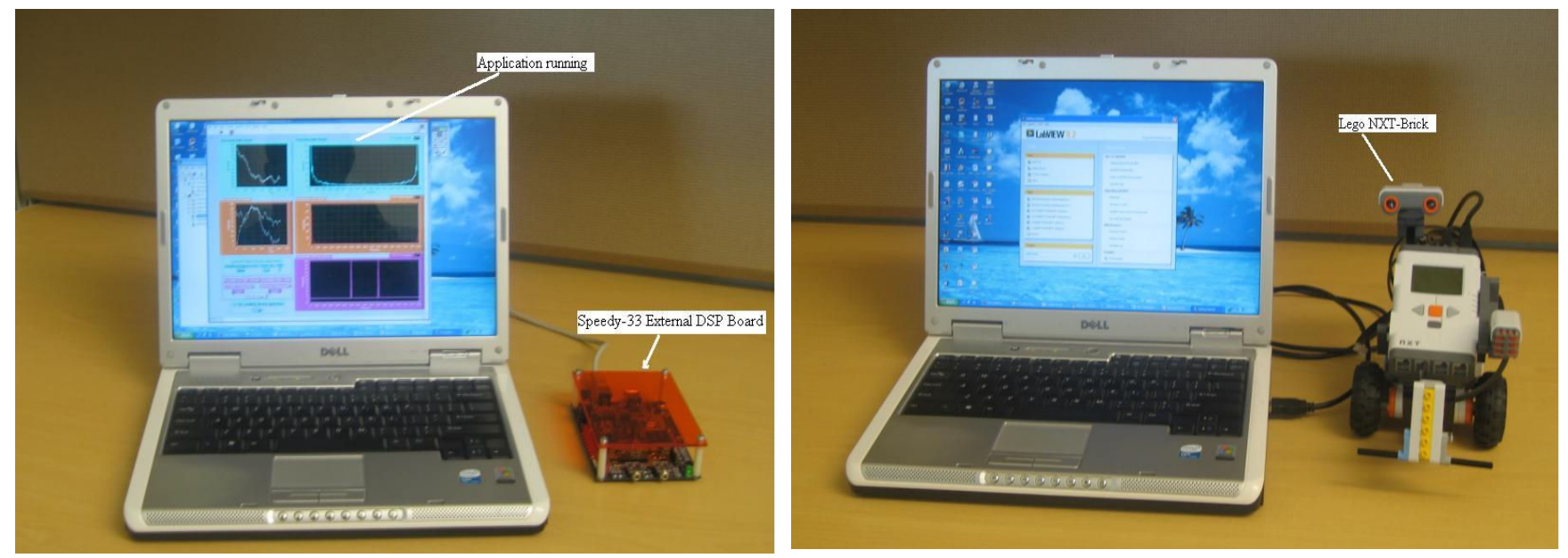

Figure 4: On the left, the NI Speedy-33 DSP board and laptop; on the right, the LEGO Mindstorms NXT Brick and laptop

\section{Sensor and Measurement Modules}

- LEGO and PIC platforms, sensors, interfacing electronics

- Done at home or in class

- Fundaments Concepts: Sensors, random and systematic errors, calibration, accuracy, repeatability, filtering, mean, cross-correlation

Sensors inherently have non-ideal characteristics, and measurements must take these characteristics into account. Students are required to characterize a set of sensors for nonideal behavior, and where appropriate, write code to correct for the errors. Measurement concepts such as precision, resolution, repeatability, hysteresis, random noise, and linearity are applied to the sensors being evaluated. One sensor package is the use of an ultrasonic device to measure distance. Students have to perform calibration measurements to convert ultrasonic readings to distance, and consider operating characteristics such as repeatability, accuracy vs. distance, environmental concerns, etc. Other sensors include sound intensity, light intensity and accelerometers. 


\section{Feedback Control Modules}

- LEGO platform, PIC platform, motors and sensors

- Done at home or in shared lab

- Concepts: Digital feedback control, system identification, programming, real-time computing, effects of time delays and sampling limitations, motor control

A simple LEGO NXT motor control experiment is shown in Figure 5. This module includes a demonstration and project. The demonstration allows students to enter gain values to the processor and observe the corresponding step response; a sample response is shown in Figure 6. This demonstration has been used in several ECE classes and one mechanical engineering class. The project requires students to identify the model and design a lead controller for it. This particular project is an update of the project described in [Heck, et al, 2004] using the LEGO Mindstorm kit. A PIC-based platform for motor control and frequency response was also developed that is a variation on the module described in [Durfee, 2005]. The new version is smaller than the original experiment and is powered from the computer USB connection rather than requiring an AC adapter. The PIC platform is cheaper than the LEGO platform and is more transparent to students in terms of parts, but is more fragile.

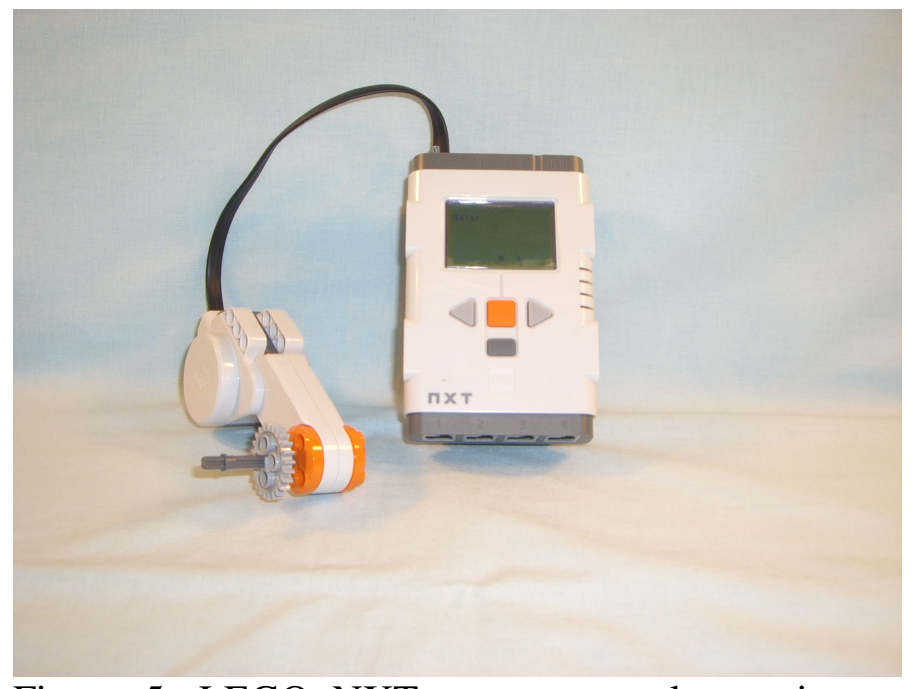

Figure 5: LEGO NXT motor control experiment. Students can enter a gain directly in the NXT processor interface and run the motor position controller with different levels of gains. They can try to turn the motor away from its set point and find that the higher the gain, the harder it is to turn the motor from its set point

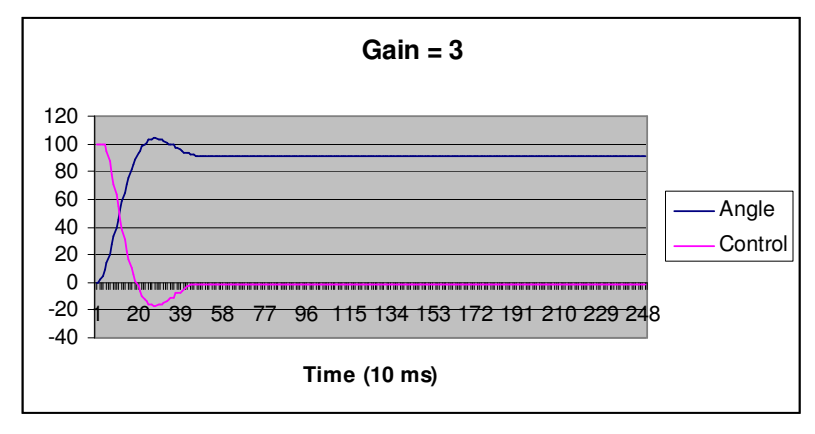

Figure 6: Motor control results for a setpoint of $90^{\circ}$ with proportional control. 


\section{Computer Modules}

- LEGO and PIC platforms

- Mode of Delivery: Done at home

- Fundamental Concepts: Operating system services, programming, I/O interfacing, measurement processing, multi-tasking

The LEGO-based projects use both the older style RCX processor and the new NXT processor. Modules on operating systems have been developed using an open source operating system (the BrickOS) for the LEGO microcontroller. An example project already used in a class in ECE was to modify the dynamic memory allocation functions (such as malloc) in order to add a linked list to identify memory locations that are free. The students had to time a benchmark program with and without their modifications. Other projects will be written that will require students to modify the task manager and the $\mathrm{I} / \mathrm{O}$ modules to accept other forms of sensor inputs and other interfaces. Another project that has been developed for the LEGO NXT kit and used in courses involves multi-tasking, in particular, programming a robot using behaviors such as escape, avoid, search, etc. These behaviors are run in parallel using tasks, where the escape behavior has the highest priority. Development is underway for computer modules using the PIC processor.

\section{Electric Power Modules}

- DC motors/generators, LED lights, AC circuit components

- Done in class, in-class demo

- Concepts: Power generation, efficiency, effects of loading

The experimental modules that involve power generation and energy efficiency use LEGO motors, light bulbs, and cheap solar panels (at the level of K-12 science kits). LEGO motors have fairly good efficiency; so it is possible to hand turn a motor so that it generates enough power for a small lamp or other motors. Students will be instructed to compare the energy they must supply to turn the generator with various loads. A particularly interesting comparison is the energy needed to light an LED versus an incandescent bulb (from a miniature Christmas tree string). An LED and the incandescent bulb produce about the same intensity of light, but the amount of energy needed to turn the generator is remarkably different. LEDs are much more efficient, meaning that it is almost effortless to turn the generator by hand. The efficiency of LEDs over incandescent bulbs has made solid state lighting a very important research topic in the past few years. Sample current and voltage curves for lighting the LED and the incandescent bulbs can be supplied to the students to compute the power for each type of light. Another experiment, shown in Figure 7, involves a solar panel supplying power to a potentiometer. The current through the potentiometer is measured with a cheap ammeter permanently mounted in the experiment, and a digital voltmener measures the voltage across the potentiometer. The students can turn the potentiometer and compute the current-voltage curves. Another option is for students to compute the power generated at various distances from a light source and then plot the power as a function of distance from the source. This experiment costs on the order of $\$ 35$ (not including the digital voltmeter, which is a stock item in the laboratories). 

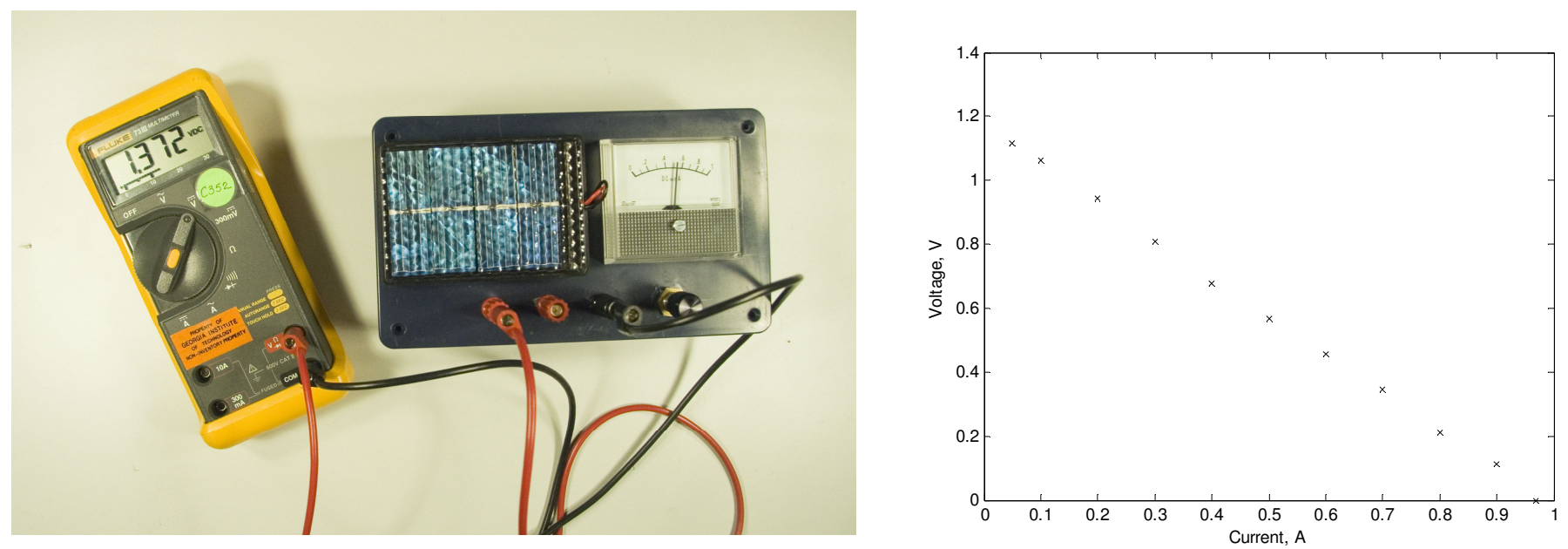

Figure 7: Solar panel experiment to measure I-V curves, on left, with resulting I-V curve, on right.

\section{Robotics}

- $\quad$ LEGO kits, Amigobot mobile robots

- Mode of Delivery: Done at home or in shared lab

- Fundamental Concepts: Programming, sensors, communications, real-time control, mechanics, hierarchical control

The experimental modules are designed to allow students to grasp the concepts of information flow from sensing to thinking to acting in autonomous systems. The LEGO NXT processor is capable of multi-tasking and is used to program behaviors for mobile robots. Figure 8 shows a student project where two robots communicated via a Bluetooth connection and performed coordinated tasks. The Amigobots include a number of sensor devices, integrated low-level control, and the ability to program new behaviors. The Amigobot platform is used to demonstrate and understand concepts such as the need for system adaptation in dynamic environments, the motivation for intelligent control, and the effect of sensor failure and noise, among others. In addition, for in-lab projects, students experiment with integrating high-level and low-level control methodologies for real-world implementation. The Amigobots were used in Spring 2007 in a robotics course. Most of the student projects added a camera to the Amigobots to add vision capabilities and programmed the robots for a variety of tasks such as following a certain color, avoiding obstacles, and searching for a particular object. One of the term projects can be seen in Figure 9. The student teams first used simulators such as Player/Stage or MobileSim to troubleshoot their control programs and then used the same programs on the actual platforms. The robotics modules can be used in systems and controls courses, robotics courses, and programming courses. 


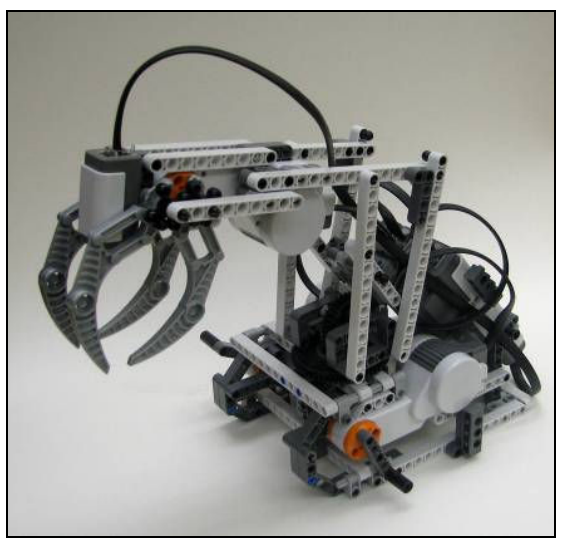

Figure 8: Term project completed with the LEGO NXT, this robotic arm was interfaced with another LEGO NXT mobile robot to perform coordinated tasks using wireless communications.

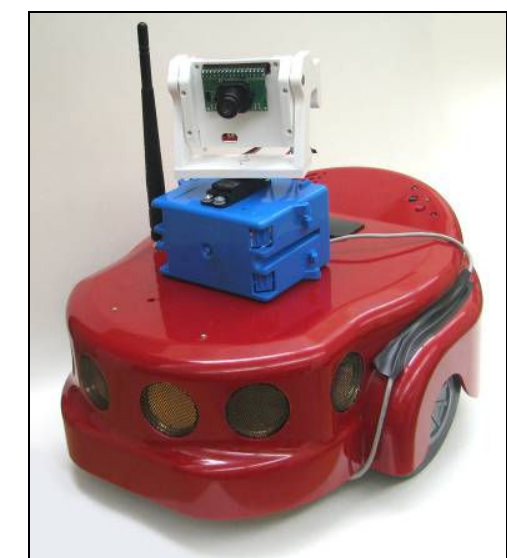

Figure 9: Term project completed with the Amigobot. The cameral was used for obstacle avoidance and path following.

These modules can be used in a variety of undergraduate ECE courses at our institution, none of which has an existing experimental component to it. The experimental modules are designed to compliment the material covered in the class.

\section{Assessment}

The goal of this instructional approach is to improve student understanding of fundamental and complex engineering concepts with the integration of the experiments in selected courses. A summative and formative assessment model will be used. To this end, both quantitative and qualitative data will be collected starting in the Spring semester of 2008.

The primary expected outcome is that student achievement on tests will be higher in classes that incorporate the hands-on modules. Student achievement will be evaluated using a rigorous model with control and experimental groups. An analysis of student test performance on those questions that correspond to the material covered with hands-on experiment will be compared to the control group that utilizes the traditional instructional approach without the supplemental experiments. Appropriate statistical techniques will be used to compare the performance of the student groups. In addition, students from the experimental group (those in classes using the hands-on experiment) and from the control group (those in classes using the traditional instruction) will be compared using selected demographic variables in order to consider other possible explanations for differences in test performance. To achieve comparable control groups, the implementation schedule will maximize opportunities for the same instructors to use the modules in one course offering and then to use the traditional method of teaching the material in subsequent or concurrent class offerings. In some instances, other scheduling constraints may prohibit the direct comparability of the classes and introduce confounding variables such as instructor variability or class size, but all efforts to limit these cases will be made. If and when this occurs, the pure control group model cannot be used and efforts to limit variability between comparison groups will be done.

Another expected outcome is that students using the modules will respond favorably to the course material and to the integration of hands-on experiments in their classes. Student surveys 
will be used to better understand the impact of the experiments on attitudes about course content, relevance of material, application of concepts, interest and motivation about course content, and overall quality of class experience. This data will enhance understanding about the broader impact of using hands-on instructional methods. Both assessment tools, comparative analysis of student performance and student attitudinal data, will address the effectiveness of the hands-on experiments to enhance student learning.

The third expected outcome is to effectively use the modules in a variety of teaching forums. Formative research will be conducted in the second year in order to improve implementation and instructional methods. Initially, students in the classes will be asked to provide feedback about the clarity of instructions, procedures and objectives, integration of the experiment in the course content, learning style preferences, and when appropriate helpfulness of the on-line components. Focus groups and student surveys will be used for this assessment component. Once the modules are adapted and utilized for other user groups, survey materials will also be modified for these other purposes. Thus, systematic feedback will be collected and used to improve delivery and implementation practices for the multiple user groups with the intent of developing "best practices" guidelines for implementation and instruction of each module.

To improve implementation procedures and adaptability of the modules, productivity measures will be collected that are relevant to scheduling of shared resources (in this case, the experimental platforms). A tracking system for wait time, throughput, and utilization will be required in order to monitor and quantify demand for these shared resources. Resulting guidelines will be integrated in the "best practices" materials for use by other schools and groups.

\section{Summary}

The work funded under the NSF CCLI grant develops several portable experiments and related web modules for each experiment, establishment of procedures and logistics to incorporate these experiments into the ECE curriculum at Georgia Tech, introduction of these experimental modules and procedures into existing ECE and ME classes, and assessment of the developed experimental modules and procedures. We have a laboratory that houses these portable experiments and can be used on a short-term basis by individual classes to run experiments. This laboratory serves as a place to hold sessions on hands-on demos to visiting high school groups in addition to serving as a basis for camps for high school and middle school students.

A goal of the experimental modules is to make them as accessible as possible to as wide a range of instructors as possible. These experimental modules are being designed primarily for faculty who do not want to spend a lot of money for experiments or time developing, building and maintaining experiments. Moreover, the goal of these experiments is to help teach fundamental concepts, not laboratory procedures. Therefore, hands-on demos must be easy for students to use. We want the experiments to supplement material in existing courses and not require faculty to change their standard assessment methods, such as in-class tests. Therefore, the supplemental web material for each experiment contains a tutorial for students on the fundamental concepts being taught and an online quiz for them that gives representative questions on the material that might be found on a standard exam. Five of the modules have been used in classes already and assessment will begin in Spring 2008. 


\section{Acknowledgement}

Sponsor: NSF CCLI Program, Russell Pimmel Project Director, project number 0618645. The authors appreciate the help of Edgar Jones, James Steinberg, and Jennifer Garcia in building the experimental platforms.

[1] Dua, R., Seiffertt, J.E., Blaha, B., Gupta, K., Satagopan, V., Stanley, J.R., Beetner, D., and Wunsch, D.C., 2005, "Hands-On Projects and Exercises to Strengthen Understanding of Basic Computer Engineering Concepts," Proceedings of the American Society of Engineering Education Annual Conference \& Exposition, Portland, OR.

[2] Durfee, W., Li, P., and Waletzko, D., 2005, “At-Home System and Controls Laboratories," Proceedings of the American Society of Engineering Education Annual Conference \& Exposition, Portland, OR.

[3] Heck, B.S, Clements, N. S., and Ferri, A.A., 2004, "A LEGO Experiment for Embedded Control System Design,” IEEE Control Systems Magazine, Oct. 2004, pp. 61-64.

[4] Hendricks, R.W., Lai K.M., and Webb, J.B., 2005,"Lab-in-a-Box: Experiments in Electronic Circuits That Support Introductory Courses for Electrical and Computer Engineers," Proceedings of the American Society of Engineering Education Annual Conference \& Exposition, Portland, OR.

[5] Litwhiler, D.H., Lovell, T.D., 2005, "Acoustic Measurements Using Common Computer Accessories: Do Try This at Home," Proceedings of the American Society of Engineering Education Annual Conference \& Exposition, Portland, OR.

[6] Broberg, H.L., and Thompson, E., 2005, "Selection of Processor, Language, and Labs in Introductory Microprocessor/Microcontroller Courses," Proceedings of the American Society of Engineering Education Annual Conference \& Exposition, Portland, OR.

[7] Williams, A.B., 2003, "The Qualitative Impact Of Using LEGO MINDSTORMS Robots To Teach Computer Engineering," IEEE Transactions on Education, v 46, n 1, Feb. 2003, p206.

[8] González, O., Rodríguez, M., Ayala, A., Hernández, J., Rodríguez, S., 2004, “Application of PIC's and Microcontrollers in the Measurement and Control of Parameters in Industry," International Journal of Electrical Engineering Education, available online http://www.findarticles.com/p/articles/mi_qa3792/is_200407/ai_n9470066.

[9] Kamen, E.W.,and Heck, B.S., 2000, Fundamentals of Signals and Systems Using MATLAB and the Web, Prentice Hall, $2^{\text {nd }}$ ed.

[10] FitzSimmons, J. and Hug, B., 2005, "Web Modules: New Toys for Engineering Students to Play With," Proceedings of the American Society of Engineering Education Annual Conference \& Exposition, Portland, OR.

[11] McClellan, J.H., Shafer, R.W., and Yoder, M.A., 1997, DSP First: A Multimedia Approach, Prentice Hall Publishers. 